\title{
2847. Development of a finger like multi-joint articulated surgical retractor for use in endoscopic surgery
}

\author{
Ikuo Yamamoto ${ }^{1}$, Keiko Kishikawa ${ }^{2}$, Yoshihiro Kondo ${ }^{3}$, Murray Lawn ${ }^{4}$, Takeshi \\ Nagayasu $^{5}$, Naoya Yamasaki ${ }^{6}$, Keitaro Matsumoto ${ }^{7}$ \\ 1,2, ${ }^{3}$ Department of Mechanical Science, Nagasaki University Graduate School, Nagasaki, Japan \\ ${ }^{4}$ Medical-Engineering Hybrid Professional Development Program, Nagasaki University Graduate School \\ of Biomedical Sciences, Nagasaki, Japan \\ ${ }^{5,6,7}$ Department of Surgery, Nagasaki University Graduate School of Biomedical Sciences, \\ Nagasaki, Japan \\ ${ }^{4}$ Corresponding author \\ E-mail: ${ }^{1}$ iyamamoto@nagasaki-u.ac.jp, ${ }^{2}$ keiko.kishikawa.32@gmail.com, ${ }^{3} y$-kondo@nagasaki-u.ac.jp, \\ 4lawnmj@nagasaki-u.ac.jp, ${ }^{5}$ nagayasu@nagasaki-u.ac.jp, ${ }^{6}$ ynaoya@nagasaki-u.ac.jp, \\ ${ }^{7}$ kmatsumo@nagasaki-u.ac.jp
}

Received 14 June 2017; received in revised form 12 October 2017; accepted 28 October 2017 DOI https://doi.org/10.21595/jve.2017.19326

Check for updates

\begin{abstract}
The authors have developed an articulated medical instrument which assists with the movement of and maintenance of the position of such as a lung during surgery to assist providing improved viewability and providing the necessary space required to work efficiently and effectively. Conventionally holding such as, a lung in a given position is achieved by arranging gauze in an appropriate configuration with the aid of a simple shaft like surgical instrument, however for various reasons the gauze requires constant replacement and rearrangement resulting in significant surgical inefficiency. By using an articulated mechanism that mimics the functionality of fingers, we have developed a surgical device that inherently provides greater mechanical stability and does not require the use of gauze, thus enabling more efficient surgery. The prototyping process was assisted with the use of 3D-CAD to simulate static and dynamic conditions and a 3D-printer to confirm the concept and then a working prototype was created. In order to validate the dynamic functionality of this surgical instrument, it was tested on a porcine lung which closely approximates human lungs by a clinical doctor of thoracic surgery. The result was that the developed prototype articulated mechanism was able to stably move and hold the lung in position during surgery thus confirming the dynamic efficacy of the mechanism.
\end{abstract}

Keywords: surgical retractor, articulated mechanism, endoscopic surgery, 3D-CAD, 3D printer, dynamic stability.

\section{Introduction}

In recent years, the number of cases of endoscopic surgery being used as a minimally invasive treatment has increased significantly $[1,2]$, furthermore an ever-widening range of technologies are being employed to meet this demand [3]. However endoscopic surgery presents many challenges, one of the significant difficulties is the high level of specialized skills required by the surgeon. Specifically working in a confined space with limited view-ability, the dynamic task of retracting soft, yet delicate, semi-fluid-like organs to ensure unimpeded viewing is essential for safe and efficient surgery. In regard to thoracoscopic surgery this may involve positioning part of a lung during surgery. At the time of writing typically a surgical instrument ( $5 \mathrm{~mm}$ forceps) is used to hold a lung segment with the assistance of gauze, the mechanism relies on the friction of relatively dry gauze. However, during surgery, the gauze becomes soaked with various body fluids including blood or cleansing solution (e.g. $\mathrm{NaCl}$ ) causing the lung to slip out of place, the gauze therefore requires constant replacement resulting in poor operating efficiency. By using an endoscopic instrument with a tip that can be articulated into a shape resembling human fingers organs can be mechanically held without the use of gauge. 
Surgical instruments intended for use in endoscopic application are required to pass through very small diameter cylindrical openings (trocars), and the standard sizes are fixed. Typically, the maximum size commonly used is nominally $10 \mathrm{~mm}(\sim 12.5 \mathrm{~mm}$ actual max.). The development of this multi-joint articulated tip mechanism is expected to contribute to the development of surgical instruments and be potentially applicable to a wide variety of clinical situations.

The purpose of this study was to develop and prototype a surgical instrument using a multi-joint articulated tip mechanism intended for use in endoscopic surgery, and to verify its effectiveness.

\section{Outline of proposed mechanism}

The proposed finger like multi-joint articulated surgical retractor is shown in Figs 1 and 2 . This retractor is designed specifically for thoracoscopic surgery. In order to pass the device through a trocar the tips are aligned and straightened allowing it to pass through a standard $10 \mathrm{~mm}$ $(12.5 \mathrm{~mm})$ port. Once the tip is inserted the tip fingers can be spread and curved as required to hold an organ, such as a lung segment. Details of the device operation are outlined in Sections 4 and 5. and functional dynamics were confirmed through a porcine experiment detailed in Section 6.

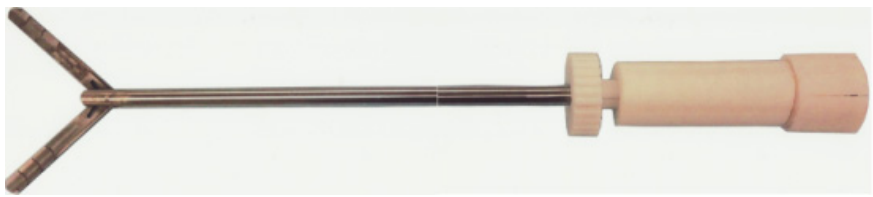

Fig. 1. Fully functional prototype retractor

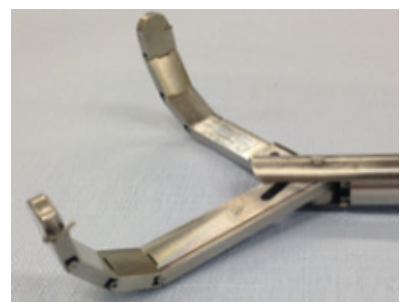

Fig. 2. Prototype retractor tip

This was designed and prototyped at the request of thoracic surgeons to provide a stable mechanism to hold lung segments in place, or more specifically out of the way to permit surgery nearby.

\section{Development process}

Fig. 3 shows the development process. The retractor was initially designed and modeled in 3D-CAD, this permitted confirmation of mechanical feasibility, operation and stress analysis. A semi-functional model was 3D printed on a Stratasys Objet260 Connex (Fig. 4) to confirm the design ergonomics with the medical staff. Specifically, physical articulation could be confirmed but actual stresses could not be applied on account of resin based 3D printers being incapable of producing the small complex parts that are required to handle the significant mechanical stress required for the retractor tip mechanism. Details regarding this rapid concept to prototype are outlined in a prior publication [4]. However, a fully functional handle and wire actuating mechanism was successfully 3D printed. The tip mechanism was then prototyped at Nagasaki University by machine specialists and the fully functional retractors were evaluated by medical staff and the functionality and operating dynamics were confirmed during an operation on a porcine lung which closely resembles human lungs. 


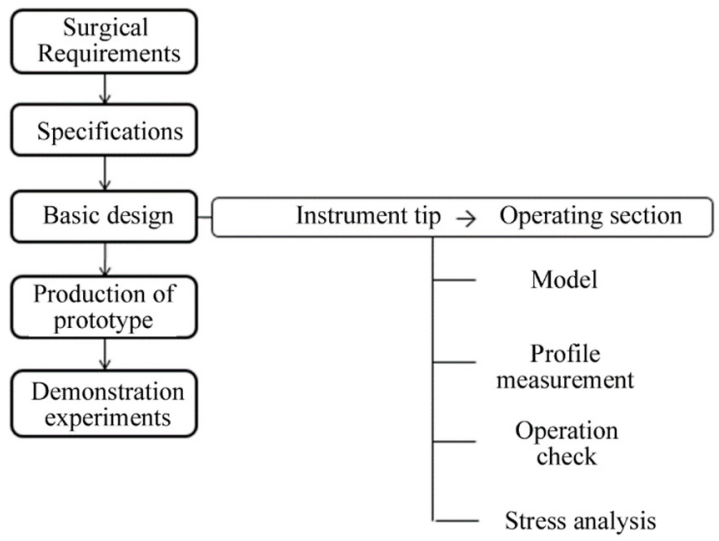

Fig. 3. Development flow

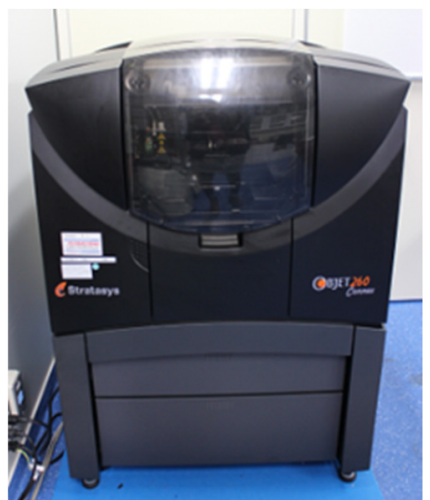

Fig. 4. 3D printer (Stratasys Objet260 Connex)

\section{Features of the multi-joint mechanism}

Fig. 5 shows the multi-joint articulated mechanism modelled in 3D-CAD and Table 1 indicates the component sizes, each finger consists of an articulated set of plates that are wire actuated from both sides to permit the bending and straightening of the fingers. The design is such that when the fingers are aligned and straightened they can pass through a 12-mm trocar. Each of the three joints provides 30 degrees of articulation providing a total curvature of 90 degrees.

Table 1. The dimensions of the surgical retractor's tip model

\begin{tabular}{|c|c|}
\hline \multicolumn{2}{|c|}{ Size } \\
\hline Plate thickness & $3 \mathrm{~mm}$ \\
\hline Plate width & $9.5 \mathrm{~mm}$ \\
\hline Plate length & $85 \mathrm{~mm}$ \\
\hline
\end{tabular}

Fig. 6(b) shows a cross section of the articulating tip mechanism. The currently used mechanism dynamics are shown in Fig. 6(a), whereby $5 \mathrm{~mm}$ forceps are used to hold a lung segment with the assistance of gauze, the mechanism relies on the friction of relatively dry gauze which inherently becomes soaked with various fluids, slipping out of place and requiring constant replacement, furthermore the contact area on account of the shape of the 5-mm shaft is poorly suited to the function of holding such as a lung compared to the ergonomics provided by the multi-joint retractor mechanism. Fig. 7 visually depicts the forces originating from the lung (due to surgical work nearby) in yellow and the counter forces provided by the current mechanism in Fig. 7(a) and (c) and the developed mechanism shown in Fig. 7(b) and (d). Clearly the developed 
mechanism provides significant improvement in dynamic stability particularly in the $X$ direction Fig. 7(d) compared to Fig. 7(c) as well as the improved operating efficiency dynamics on account of not needing to constantly replacing the gauze.

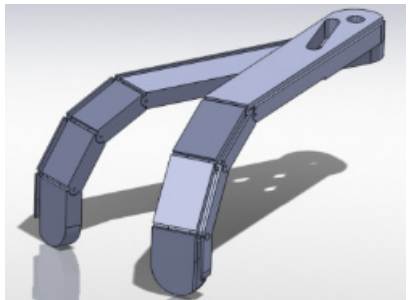

Fig. 5. Surgical retractor tip model

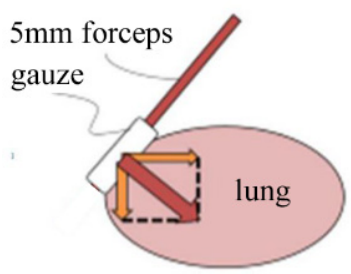

a)

Fig. 6. Comparison of conventional

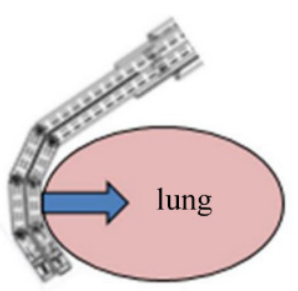

b)

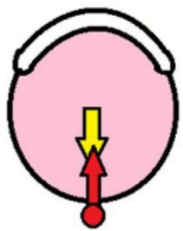

a)

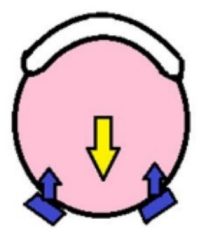

b)

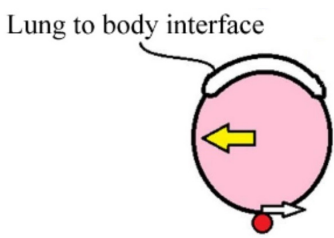

c)

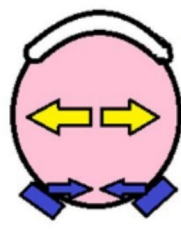

d)

Fig. 7. Comparison of single point and multipoint dynamics and multi-joint mechanism dynamics

\section{Articulation operation}

Regarding the articulating joints detailed in Fig. 8, the actuating wires are located $0.75 \mathrm{~mm}$ above and below the articulating joint center, the actuating distance (pull) is theoretically $0.13 \mathrm{~mm}$ per joint, in practice some stretch occurs in the cable depending on the pressure applied. The 3D printer used (Stratasys Objet260 Connex) successfully printed a semi-functional 3D model using actual dimensions. The 3D printer used (Stratasys Objet260 Connex) successfully printed a semi-functional 3D model using actual dimensions. Therefore, the retractor tip's bending and elongation functionality was confirmed using pulling wires using an actual size 3D printed model shown in Fig. 9.

\section{Static and dynamic stress analysis}

Using 3D-CAD software stress analysis was performed on the proposed model using SolidWorks simulation Premium software to confirm the location and magnitude of static and dynamic stresses, Figs. 10(a) overall stress on a single cable and Figs. 10(b) close up of single joint. This then provided the data on which to base the choice of materials to be used for the base components. Based on this data SUS304 stainless steel $(18 \mathrm{Cr}-8 \mathrm{Ni})$ was selected for the retractor tip. Regarding the draw wires, $0.45 \mathrm{~mm}$ SUS304 Stainless steel wire rope was selected providing a $b / s$ (breaking strain) of $16 \mathrm{~kg}$. Based on this and the previous specifications a maximum force of approximately 400 grams may be applied on each tip, giving a net maximum of 800 grams or 
approximately $8 \mathrm{~N}$. Surgical staff indicated that this would provide an acceptable dynamic safety margin in the case of moving around and positioning lung segments during an operation. If this was to be used to stabilize heavier organs the number of actuating cables would need to be increased on the lower (bending actuation) layer.

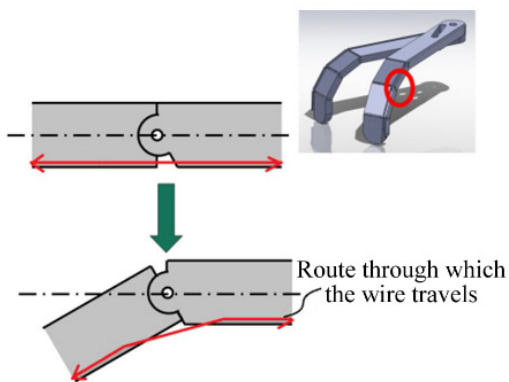

Fig. 8. Joint detail

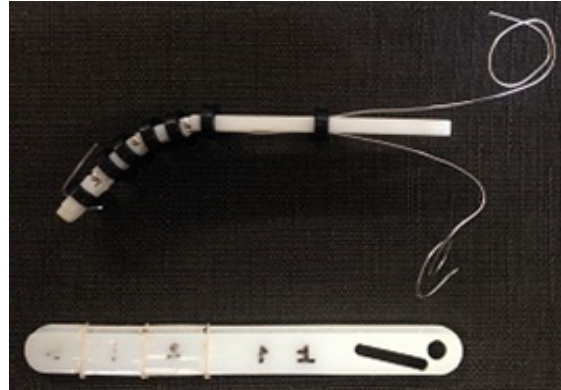

Fig. 9. 3D printed functional test

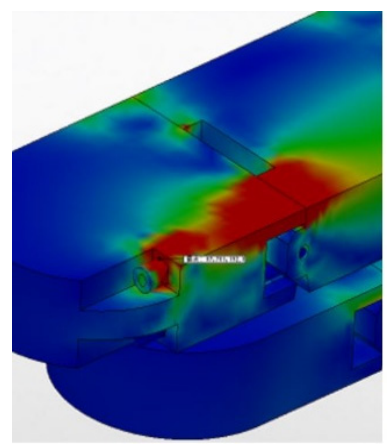

b)

Fig. 10. a) Simulated stress analysis - verification of safety margins, b) close up of single joint

\section{Use of developed retractor in porcine experiment - confirmation of operating dynamics}

The completed fully functional prototype retractor is shown in Figs. 1 and 2. The center main part of the handle is the main handle, the section to the left of the handle in Fig. 1 adjusts the opening angle of the tips (shown open) by rotation. The end section to the right of the handle in Fig. 1 adjusts the tip's flexion (or straightening) by rotation. The total length is about $500 \mathrm{~mm}$ and all metal parts are made using SUS304 stainless steel. The handle and internal control mechanisms were 3D printed from ABS resin.

Fig. 11(a) and (b). shows the retractors being used to hold part of a porcine lung in place in preparation for surgery on the target tissue outlined in red. In Fig. 11(a) the target tissue is obstructed by a lung segment, in Fig. 11(b) the lung segment is retracted, and the target tissue area is clearly visible and ready for surgery. The fully functional prototype retractors were successful in carrying out this role thus validating the mechanism's functionality and dynamics in thoracic surgery. By using the multi-joint finger like articulated mechanism, the surgery was able to be carried out in an efficient and effective manner without the need for use of gauze as the mechanisms dynamics are such that they can be inherently adapted to the shape of the organ that it needs to support.

Regarding evaluation of prototype laparoscopic instruments in general the current standard is the "Rosser station test" [5], handling ergonomics in regard to dynamics confirmed by EMG [6], however in the case of retractors, mechanical simulations and actual tests in regard to fitness for given tasks are more typical [7]. 


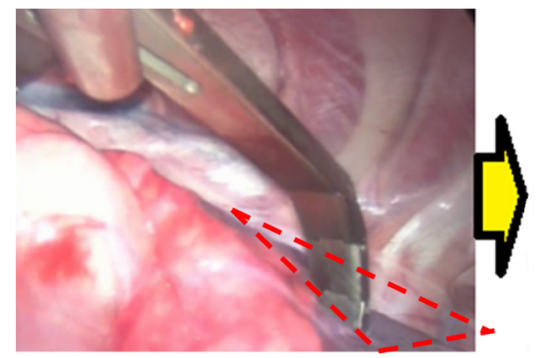

a)

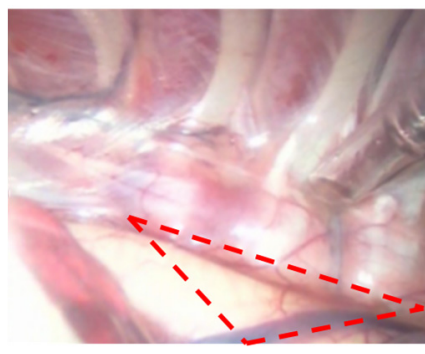

b)

Fig. 11. a) Target tissue obstructed by lung segment,

b) lung segment retracted-target tissue made accessible

This research was overseen by Ikuo Yamamoto (Engineering) and Takeshi Nagayasu (Medical). The research was carried out by and written up by Keiko Kishikawa and Yoshihiro Kondo with the assistance of the other authors. Naoya Yamasaki facilitated the porcine experiment and Keitaro Matsumoto the initial 3D printing. The full metal forceps were arranged by Ikuo Yamamoto. All authors worked together on the conceptual design of the forceps. The English paper was checked, corrected and the revised by Murray Lawn.

\section{Conclusions}

Despite the stringent dimensional limitations required for use of a standard laparoscopic port $(\sim 12 \mathrm{~mm})$ the authors developed and prototyped a surgical retractor using a multi-joint articulated mechanism and a handle with the necessary control mechanism built into it.

The functionality of this retractor was verified in a porcine experiment on lungs similar to those of human lungs. It is expected that this mechanism could have application in a wide range of clinical applications. While clinical trials are planned, a number of issues need to be dealt with, namely protection against pinching tissue in the linkages, and the aspect of sterilization.

The development of these receptors was assisted with the use of a 3D printer for both initial functional prototyping and for prototyping of the fully functional handle and the control mechanism that is built into the handle. Finally, the current mechanism would most likely be classified as suitable for single use application (disposable) due to the complexity of the tip mechanism making sterilization difficult, therefore modifications will be required to make the mechanism easier to sterilize and therefore reusable.

\section{Acknowledgements}

A part of this study was supported by the Medical Engineering Hybrid professional development program staff. The authors would like to express their sincere gratitude to Mr. Naoto Matsuo, Mr. Nobuo Kakinoki, Nagasaki University and related personnel in this research and development.

\section{References}

[1] Hashimoto Daijyo Current problems and its solutions in minimally invasive surgery. Modern Media, Vol. 50, Issue 12, 2004, p. 7.

[2] Khatuja Ritu, Jain Geetika, Mehta Sumita, Arora Nidhi, Juneja Atul, Goel Neerja Changing trends in use of laparoscopy: a clinical audit. Minimally Invasive Surgery, 2014, https://doi.org/10.1155/2014/562785.

[3] Vitiello Valentina, Su Lee Lin, Cundy Thomas P., Yang Guang Zhong Emerging robotic platforms for minimally invasive surgery. IEEE Reviews in Biomedical Engineering, Vol. 6, 2013, p. 111-126.

[4] Yamamoto Ikuo, Ota Ren, Zhu Rui, Lawn Murray, Ishimatsu Takakazu, Nagayasu Takeshi, Yamasaki Naoya, Takagi Kazunori, Koji Takehiko Research on seamless development of surgical 
instruments based on biological mechanisms using CAD and 3D printer. Journal Bio-Medical Materials and Engineering, Vol. 26, 2015, p. 341-345.

[5] Mary Frecker I., Jeremy Schadler, Randy Haluck S., Kristin Culkar, Ryan Dziedzic Laparoscopic multifunctional instruments: design and testing of initial prototypes. Journal of the Society of Laparoendoscopic Surgeons, Vol. 9, 2005, p. 105-112.

[6] Matern U., Kuttler G., Giebmeyer C., Waller P., Faist M. Ergonomic aspects of five different types of laparoscopic instrument handles under dynamic conditions with respect to specific laparoscopic tasks: an electromyographic-based study. Surgical Endoscopy, Vol. 18, Issue 8, 2004, p. 1231-41.

[7] O'shea Conor, Kelliher Denis, Andrews Emmet, O'ríordáin Mícheál, O'shea Michael, Power Timothy, Murphy Pádraig Cantillon Design, development and evaluation of an inflatable retractor for atraumatic retraction in laparoscopic colectomy. Annals of Biomedical Engineering, Vol. 42, Issue 9, 2014, p. 1942-1951.

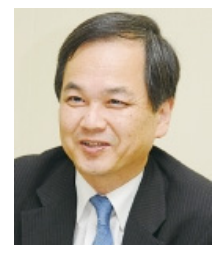

Ikuo Yamamoto received a Dr.Eng. in 1994 at Kyushu University, Japan and became a Professor at Kyushu University in 2005, the University of Kitakyushu in 2007, and Nagasaki University, Japan in 2013. Also, he became a GlobalScot member of Scotland, UK in 2017. His current research interests include robotics, mechatronics, and IoT.

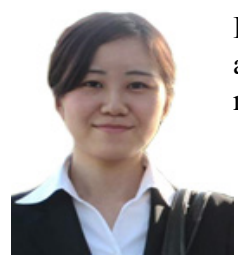

Keiko Kishikawa received a M.Eng.Sc. at Nagasaki University in 2017, Japan. She works at Toyota Motor Corp., Japan. Her current research interests include robotics and mechatronics.

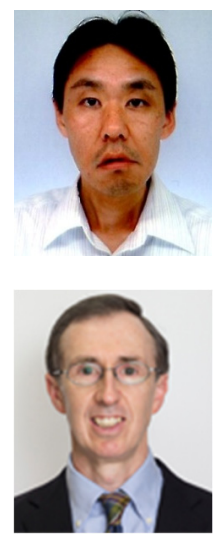

Yoshihiro Kondo received a Ph.D. degree in mechanical and control engineering at Nagasaki University, Japan, in 2002. Now he works in the Engineering Department at Nagasaki University as a technician. His current research interests include control, dynamics, and medical engineering.

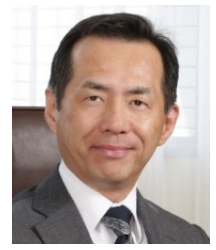

Murray Lawn received a Ph.D. degree in mechanical and control engineering at Nagasaki University, Japan, in 2003. Now he works as the coordinator for the Medical Engineering Hybrid Program at Nagasaki University. His current research interests include medical engineering and the cultivating of the next generation of medical engineers.

Takeshi Nagayasu received a Ph.D. degree at the Nagasaki University School of Medicine, Japan, in 1996. Now he works as a Professor of surgery and as the Dean of Nagasaki University School of Medicine. His current research interests include medical engineering, surgical oncology, and regeneration of respiratory organs. 
2847. DEVELOPMENT OF A FINGER LIKE MULTI-JOINT ARTICULATED SURGICAL RETRACTOR FOR USE IN ENDOSCOPIC SURGERY.

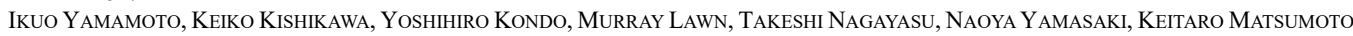

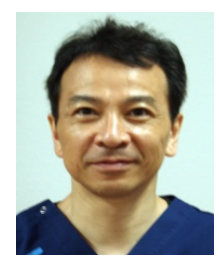

Naoya Yamasaki received an M.D. at the Oita University School of Medicine, Japan, in 1989. Now he works as an Associate Professor of Surgical Oncology of Nagasaki University School of Biomedical Sciences. His current research interests include medical engineering, lung transplantation, and regeneration of respiratory organs.

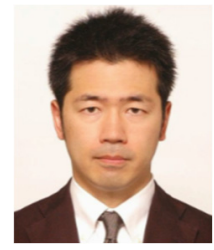

Keitaro Matsumoto received an M.D. in 1996 at the Kumamoto University School of Medicine in 1996 and a Ph.D. at the Nagasaki University Graduate School in 2009. Now he works in the Division of Surgical Oncology, Department of Translational Medical Sciences, Nagasaki University. His current research interests include medical engineering, thoracic and endoscopic surgery and regenerative medicine. 